\title{
A High Order Accurate Difference Scheme for Complex Flow Fields
}

\author{
Dexun Fu and Yanwen $\mathrm{Ma}^{1}$ \\ LNM, Institute of Mechanics, Chinese Academy of Sciences, Beijing 100080, China
}

Received October 1, 1994; revised April 26, 1996

\begin{abstract}
A high order accurate finite difference method for direct numerical simulation of coherent structure in the mixing layers is presented. The reason for oscillation production in numerical solutions is analyzed. It is caused by a nonuniform group velocity of wavepackets. A method of group velocity control for the improvement of the shock resolution is presented. In numerical simulation the fifthorder accurate upwind compact difference relation is used to approximate the derivatives in the convection terms of the compressible N-S equations, a sixth-order accurate symmetric compact difference relation is used to approximate the viscous terms, and a three-stage $\mathrm{R}-\mathrm{K}$ method is used to advance in time. In order to improve the shock resolution the scheme is reconstructed with the method of diffusion analogy which is used to control the group velocity of wavepackets. (c) 1997 Academic Press
\end{abstract}

\section{INTRODUCTION}

Great achievement has been reached in direct numerical simulation of the coherent structure in flow fields and turbulence. Good results which quantitatively agree well with experiments have been obtained for some typical incompressible flow models, and some new mechanisms have been observed. For many practical applications it requires understanding compressible turbulent flow. Although direct numerical simulation of compressible turbulence just started in recent years, it developed very fast [1-3] and has become one of the important tools for the study of turbulence. The compressible mixing layer is a good model for the study of compressible turbulence [4-8]. The key point for correctly simulating the unsteady structure of the complex flow fields with a range of scales is that the method can capture well the vortices with small scales and shocks. The method of simulation must be highly accurate and can give high resolution of the shocks. Spectral/pseudospectral methods suffer deficiency in the treatment of discontinuities which occur in the supersonic flow fields, and the finite difference methods are more popular. The high order accurate centered schemes offer little to no advantage over spectral methods in the presence of unresolved high gradients such as shocks. The upwind-biased schemes are better because the spurious waves can be suppressed, but there

\footnotetext{
${ }^{1}$ E-mail: fud@ecs.imech.ac.cn.
}

are still oscillations near the shock with higher order upwind schemes and they have to be improved also. There are many second-order accurate schemes like TVD schemes which can simulate the shocks with high resolution. Many existing schemes with high resolution of the shock are complicated and expensive.

The purpose of the present paper is to present a method for better simulation of the complex flow fields with shocklets. The reason of oscillation production in numerical solutions is analyzed. It is because of nonuniform group velocity of wavepacket. In order to improve the shock resolution the group velocity must be controlled. A method of group velocity control is presented. In numerical simulation the derivatives in the convection terms of the N-S equations are discretized with the fifth-order accurate upwind compact difference approximation (UCDA5) developed in [9], the derivatives in the viscous terms are discretized with the sixth-order symmetric compact difference approximation developed by Lele in [10], and the threestage $\mathrm{R}-\mathrm{K}$ method [11] is used to advance in time. Analysis of the reason for oscillation production and scheme reconstruction for a model equation is given in Section 2, application of the method to approximate the $\mathrm{N}-\mathrm{S}$ equations is presented in Section 3, some numerical examples and computed results of the mixing layers are given in Sections 4 and 5.

\section{METHOD DEVELOPMENT}

For correct simulation of complex flow fields with a range of scales and shocks with limited computer resource it is better to use higher order schemes. Oscillations will be produced in the numerical solutions of the commonly used high order accurate schemes, including the upwind compact schemes. In the present paper UCDA5 is used to simulate the temporally growing compressible mixing layers. In order to capture shocks well the scheme is modified with the method of diffusion analogy [12].

\subsection{Model Equation and Difference Approximation}

For simplicity consider the model equation and its semidiscrete difference approximation, 


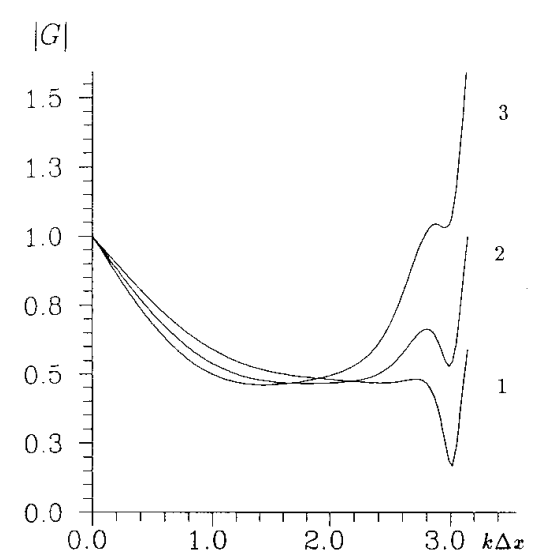

FIG. 1. Variation of amplification factor $(\mu=0$, time integration-3stage R-K, space discretization-UCDA5): $1-c(\Delta t / \Delta x)=0.49 ; 2-$ $c(\Delta t / \Delta x)=0.59 ; 3-c(\Delta t / \Delta x)=0.69$.

$$
\begin{gathered}
\frac{\partial u}{\partial t}+\frac{\partial f}{\partial x}=\mu \frac{\partial^{2} u}{\partial x^{2}}, \quad f_{u}=c,-\infty<x<\infty \\
\frac{\partial u_{j}}{\partial t}+\frac{F_{j}}{\Delta x}=\mu \frac{S_{j}}{\Delta x^{2}}
\end{gathered}
$$

where $F_{j} / \Delta x$ is an approximation of $\partial f / \partial x$ and $S_{j} / \Delta x^{2}$ is an approximation of $\partial^{2} u / \partial x^{2}$. In the present computation the first derivative is approximated by the UCDA5 which is expressed as

$$
\frac{3}{5} F_{j}^{ \pm}+\frac{2}{5} F_{j+1}^{ \pm}=\frac{1}{60} \delta_{x}^{\mp}\left(-f_{j \pm 2}^{ \pm}+11 f_{j \pm 1}^{ \pm}+47 f_{j}^{ \pm}+3 f_{j \neq 1}^{ \pm}\right),
$$

where $F_{j}^{ \pm} / \Delta x$ is the approximation of $\partial f^{ \pm} / \partial x, f^{ \pm}=c^{ \pm} u$, $c^{+}+c^{-}=c, c^{+}>0$, and $c^{-}<0 . c^{+}$and $c^{-}$are not uniquely defined. With $c^{ \pm}=(c \pm|c|) / 2$ Eq. (2.2) has the smallest truncation error due to splitting. $\delta_{x}^{-}$and $\delta_{x}^{+}$are the 2-point backward and forward difference operators, respectively. In this section only the $c=c^{+}$case is discussed. The case $c=c^{-}$can be discussed in the same way. The semidiscrete equation (2.2) is solved with the three-stage R-K method. Variations of the amplification factor $|G|$ in stability analysis versus $\alpha=k \Delta x(0 \leq \alpha \leq \pi)$ for the case $\mu=0$, $c(\Delta t / \Delta x)=0.49,0.59$, and 0.69 are given in Fig. 1 from which the stability condition can be obtained as

$$
c \frac{\Delta t}{\Delta x} \leq 0.5900
$$

The second derivative in (2.1) can be approximated with the sixth-order accurate compact difference relation for the second derivative. For the case $f_{u}=c=0$ the stability condition is

$$
\mu \frac{\Delta t}{\Delta x^{2}} \leq 0.5234
$$

The second derivative can also be considered as a double first derivative which approximation is obtained from the sixth-order compact difference relation

$$
\frac{1}{5} F_{j+1}+\frac{3}{5} F_{j}+\frac{1}{5} F_{j-1}=\frac{14}{15} \delta_{x}^{0} g_{j}+\frac{1}{30} \delta_{x}^{0}\left(g_{j+1}+g_{j-1}\right)
$$

where $\delta_{x}^{0}=\frac{1}{2}\left(\delta_{x}^{+}+\delta_{x}^{-}\right)$. In this case $\left(f_{u}=c=0\right)$ the stability condition is

$$
\mu \frac{\Delta t}{\Delta x^{2}} \leq 0.6348
$$

The last treatment for the second derivative is used in computation of the mixing layers. The above stability results hold only for infinite/periodic domain.

With initial condition $u(x, 0)=\exp (i k x)$ the exact solution of (2.1) is

$$
u(x, t)=\exp \left(-\mu k^{2} t\right) \exp [i k(x-c t)]
$$

With initial condition $u\left(x_{j}, 0\right)=\exp \left(i k x_{j}\right)$ we have the exact solution of (2.2) as follows

$$
\begin{aligned}
u\left(x_{j}, t\right)= & \exp \left[-\mu k^{2} \frac{k_{d}^{(2)}}{(k \Delta x)^{2}} t-\frac{c k_{r}}{\Delta x} t\right] \\
& \exp \left[i k\left(x_{j}-c \frac{k_{i}}{k \Delta x} t\right)\right]
\end{aligned}
$$

For the particular schemes analytic expressions for $k_{i}, k_{r}$, and $k_{d}^{(2)}$ can be obtained. For the fifth-order upwind compact difference approximation we have $(\alpha=k \Delta x)$

$$
\begin{aligned}
k_{r} & =\frac{2+\cos (\alpha)[6 \cos (\alpha)-7-\cos (2 \alpha)]}{3[13+12 \cos (\alpha)]} \\
k_{i} & =\frac{2 \sin (\alpha)[61+15 \cos (\alpha)-\cos (2 \alpha)]}{6[13+12 \cos (\alpha)]} .
\end{aligned}
$$

For the sixth-order compact difference relation (2.5) we have

$$
k_{i}=\frac{\sin (\alpha)[14+\cos (\alpha)]}{3[3+2 \cos (\alpha)]}, \quad k_{r} \equiv 0
$$

For approximation of the second derivative with two times application of (2.5) we have $k_{d}^{(2)}=\left(k_{i}\right)^{2}$, where $k_{i}$ is obtained from (2.9c). Variations of $k_{r}$ and $k_{i}$ for some difference approximations are given in Fig. 2 from which it can be seen that the higher order accurate schemes can capture 

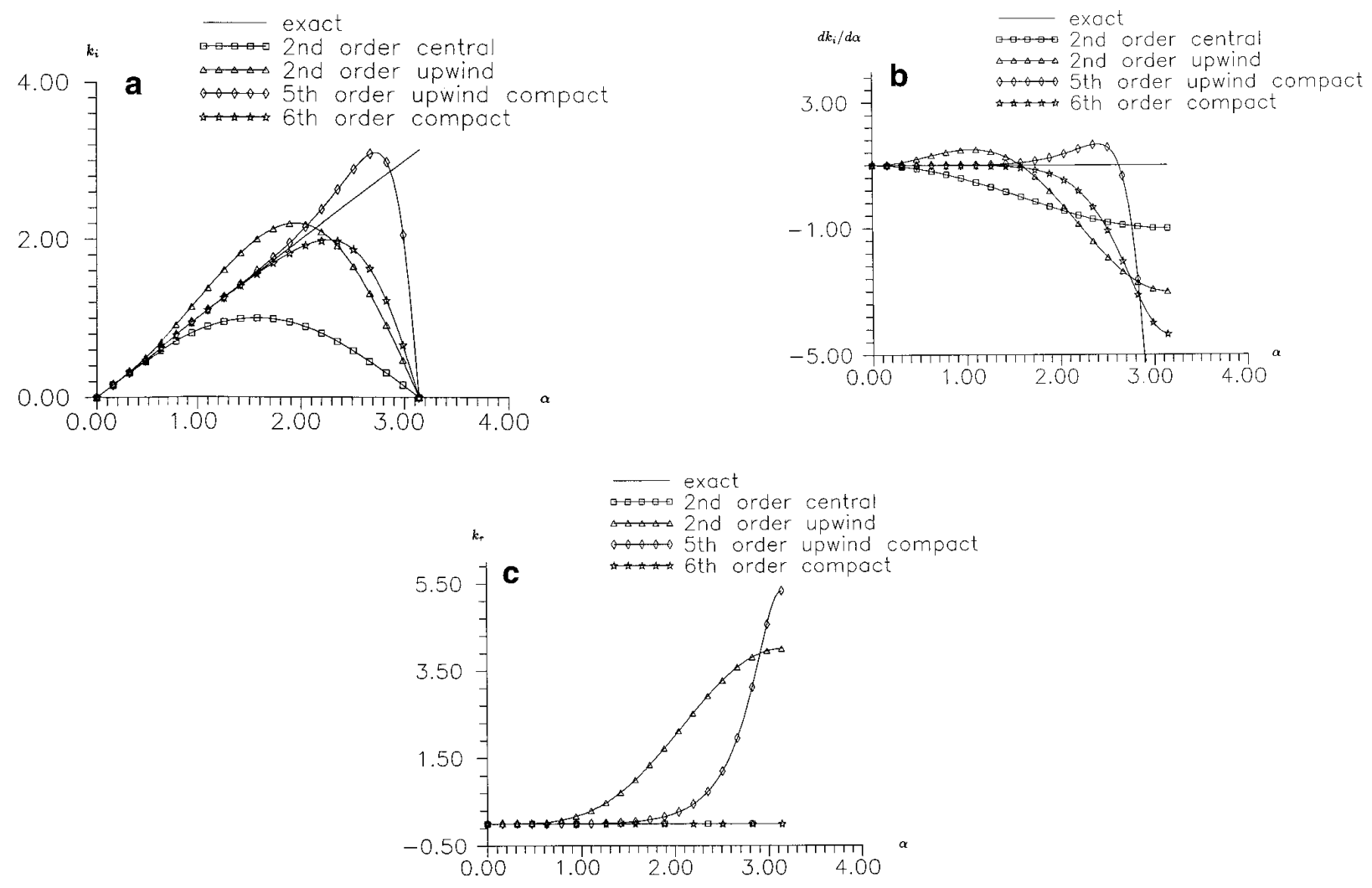

FIG. 2. a. Variation of $k_{i}$ versus $\alpha=k \Delta x$. b. Variation of $d k_{i} / d \alpha$ versus $\alpha=k \Delta x$. c. Variation of $k_{r}$ versus $\alpha=k \Delta x$.

the smaller structures better. In Fig. 2 is also given the variation of the group velocity of wavepackets which will be discussed later.

\subsection{Analysis of Oscillation Production in Numerical Solutions}

In this subsection only the case $\mu=0$ is considered because the spurious waves are produced due to approximation of the convection term. Suppose we have a N-S shock. It means that the solution is sufficiently smooth but with a very deep gradient. For this situation the Taylor series can be used everywhere. In numerical simulation of $\mathrm{N}-\mathrm{S}$ shocks with higher order accurate schemes oscillations will be produced. The reason for oscillation production is analyzed heuristically. From (2.7) with $\mu=0$ it can be seen that all the waves with different $k$ have the same phase speed and uniform group velocity of wavepackets. It means that the initial shock given at $t=0$ will keep the shape with the advance of time. From (2.8) it can be seen that $k_{i}$ is related to the phase speed in solutions of difference approximation, $k_{r}$ is related to damping of the difference solutions. $k_{i}$ and $k_{r}$ are functions of $k \Delta x$. The error in the solution of difference approximation is different for different wave numbers $k$. The accuracy of the solution depends on how well $k_{r} / \Delta x$ approaches zero and $k_{i} /(k \Delta x)$ approaches one. For the dissipative schemes $k_{r}(k \Delta x)$ damps the amplitude of the Fourier components in the numerical solutions. The damping rate is different for different $k$. Nonlinear dependence of $k_{i}$ on $k \Delta x$ leads to nonuniform group velocity which is defined as $d k_{i} / d \alpha$ in the present paper [17]. This is the reason of oscillation production in the numerical solutions. From Fig. 2 it can be seen that the Fourier components of the physical solution with lower wave numbers $k$ can be approximated well with $k_{i}$ l $(k \Delta x) \sim 1, d k_{i} / d \alpha \sim 1$, and $k_{r} / \Delta x \sim 0$. The wavepacket with lower wave numbers have group velocity very close to the physical one. When the solutions are smooth the coefficients of the Fourier components in the numerical solutions tend to zero very fast with increasing wave numbers. In this case incorrect approximation of Fourier components with high wave numbers does not influence the solution much. For the N-S shock the coefficients of the Fourier components with moderate and high wave numbers are no longer small, and they influence the behaviour of the solution. For the case $d k_{i} / d \alpha<1$ with moderate and high wave numbers the oscillations in the numerical solutions can be seen behind the shock. That is the case for the second centered and sixth-order compact difference schemes (see Fig. 2b). For the case $d k_{i} / d \alpha>1$ with moderate and high wave numbers the oscillations in numerical 
solutions can be seen in front of the shocks. For some schemes there exists $k_{o} \Delta x$ such that $d k_{i} / d \alpha>1$ for $0<$ $k \Delta x<\left(k_{o} \Delta x\right)$ and $d k_{i} / d \alpha<1$ for $k_{o} \Delta x<k \Delta x<\pi$. In this case the oscillations can be seen in both sides of the shocks. That is the case for the fifth-order accurate upwind compact scheme which has $k_{o} \Delta x=2.637$ (see Fig. 2b).

\subsection{Improvements of Shock Solutions with Group Velocity Control}

The above discussion shows that for improvement of the shock resolution the group velocity of wavepackets in the numerical solutions must be controlled. From the following two examples we can see the way for group velocity control. Consider a model equation with periodic boundary conditions

$$
\frac{\partial u}{\partial t}+\frac{\partial f}{\partial x}=\sigma c \Delta x^{2} \frac{\partial^{3} u}{\partial x^{3}}, \quad f=c u, c>0
$$

which resembles the modified equation of second-order accurate semidiscrete approximation only with the leading term. For the second-order centered difference approximation $\sigma=\frac{1}{6}$. Equation (2.10) is approximated with the following second-order semidiscrete approximation

$$
\frac{\partial u_{j}}{\partial t}+\frac{F_{j}}{\Delta x}=\sigma c \frac{\delta_{x}^{3} u_{j}}{\Delta x}-\frac{1}{2}|\sigma c| \frac{\delta_{x}^{4} u_{j}}{\Delta x}
$$

where

$$
\begin{array}{ll}
\delta_{x}^{3}=\delta_{x}^{0} \delta_{x}^{2}, & \delta_{x}^{4}=\delta_{x}^{2} \delta_{x}^{2}, \\
\delta_{x}^{2}=\delta_{x}^{+} \delta_{x}^{-}, & \delta_{x}^{0}=\left(\delta_{x}^{+}+\delta_{x}^{-}\right) / 2 .
\end{array}
$$

The right-hand side of Eq. (2.11) is obtained from oneside biased difference approximation of $\partial^{3} u / \partial x^{3}$ according to the sign of $\sigma c$ in (2.10). The following two approximations are considered:

$$
\begin{aligned}
& C N T: F_{j}=\delta_{x}^{0} f_{j} \\
& U P W: F_{j}=\delta_{x}^{f} f_{j}=\frac{1}{2} \delta_{x}^{-}\left(3 f_{j}-f_{j-1}\right) .
\end{aligned}
$$

With the initial condition $u=\exp \left(i k x_{j}\right)$ the exact solution of Eq. (2.11) has the form (2.8), but with $k_{d}^{(2)}=0$,

$$
\begin{aligned}
& k_{i}=\sin (\alpha)[1+2 \sigma(1-\cos (\alpha))] \\
& k_{r}=2|\sigma|[1-\cos (\alpha)]^{2}
\end{aligned}
$$

for CNT, and

$$
\begin{aligned}
& k_{i}=\sin (\alpha)[2-\cos (\alpha)]+2 \sigma \sin (\alpha)[1-\cos (\alpha)] \\
& k_{r}=[1-\cos (\alpha)]^{2}+2|\sigma|[1-\cos (\alpha)]^{2}
\end{aligned}
$$

for UPW. The following group velocity (divided by $c$ ) can be obtained from (2.14a) and (2.15a):

$$
\begin{aligned}
C N T: \frac{d k_{i}}{d \alpha}= & \cos (\alpha)+2 \sigma\left\{[\sin (\alpha)]^{2}\right. \\
& +\cos (\alpha)[1-\cos (\alpha)]\} \\
U P W: \frac{d k_{i}}{d \alpha}= & \cos (\alpha)[2-\cos (\alpha)]+[\sin (\alpha)]^{2} \\
& +2 \sigma\left\{\cos (\alpha)[1-\cos (\alpha)]+[\sin (\alpha)]^{2}\right\}
\end{aligned}
$$

The variations of $k_{i}, d k_{i} / d \alpha$, and $k_{r}$ for schemes CNT and UPW versus $\alpha=k \Delta x$ with different values of the parameter $\sigma$ are given in Figs. 3 and 4. From the figures it can be seen that in a wide range of $k \Delta x$ the group velocity can be controlled by the parameter $\sigma$. With proper choice of the parameter $\sigma$ we can get the group velocity higher or lower than the group velocity of the wavepackets of an exact solution in a wide range of the wave numbers. From Fig. $3 \mathrm{~b}$ and Fig. $4 \mathrm{~b}$ it also can be seen that the wavepackets with high wave numbers for the considered schemes always have lower group velocity, even negative for positive $\sigma$. From Fig. 3c and Fig. $4 c$ we see that dissipation in the range of high wave numbers is very large. So the oscillations produced by the wavepackets with higher wave numbers can be suppressed by the large dissipation. For group velocity control the parameter $\sigma$ is defined as

$$
\sigma=\sigma_{0} \operatorname{sign}\left(\frac{\partial^{2} u}{\partial x^{2}} / \frac{\partial u}{\partial x}\right), \quad \sigma_{0}>0 .
$$

As we know, in front of the shock $\left(\partial^{2} u / \partial x^{2}\right) /(\partial u / \partial x)<0$, and we have slower group velocity with $\sigma<0$ defined in (2.17) for all wave numbers. Behind the shock $\left(\partial^{2} u / \partial x^{2}\right) /$ $(\partial u / \partial x)>0$ we have $\sigma>0$. In this case the group velocity of wavepackets in a wide range of wave numbers is higher.

From the above examples we see that a given scheme can be reconstructed according to the modified equation so that in a wide range of wave numbers the Fourier components in the numerical solutions have a faster group velocity of wavepackets behind the shock and a slower group velocity in front of the shock. Consider the more general case. Suppose we have the following modified equation:

$$
\frac{\partial u}{\partial t}+\frac{\partial f}{\partial x}=\Delta x \sum_{m} C_{m} \frac{\partial^{m} f}{\partial x^{m}}, \quad m>1
$$

For the UCDA5 $C_{2}=C_{3}=C_{4}=C_{5}=0, C_{6}=$ $12 / 5\left(\Delta x^{4} / 6\right.$ !). Equation (2.18) can be written

$$
\frac{\partial u}{\partial t}+\frac{\partial f}{\partial x}=\Delta x \frac{\partial}{\partial x} \tilde{\mu} \frac{\partial u}{\partial x},
$$



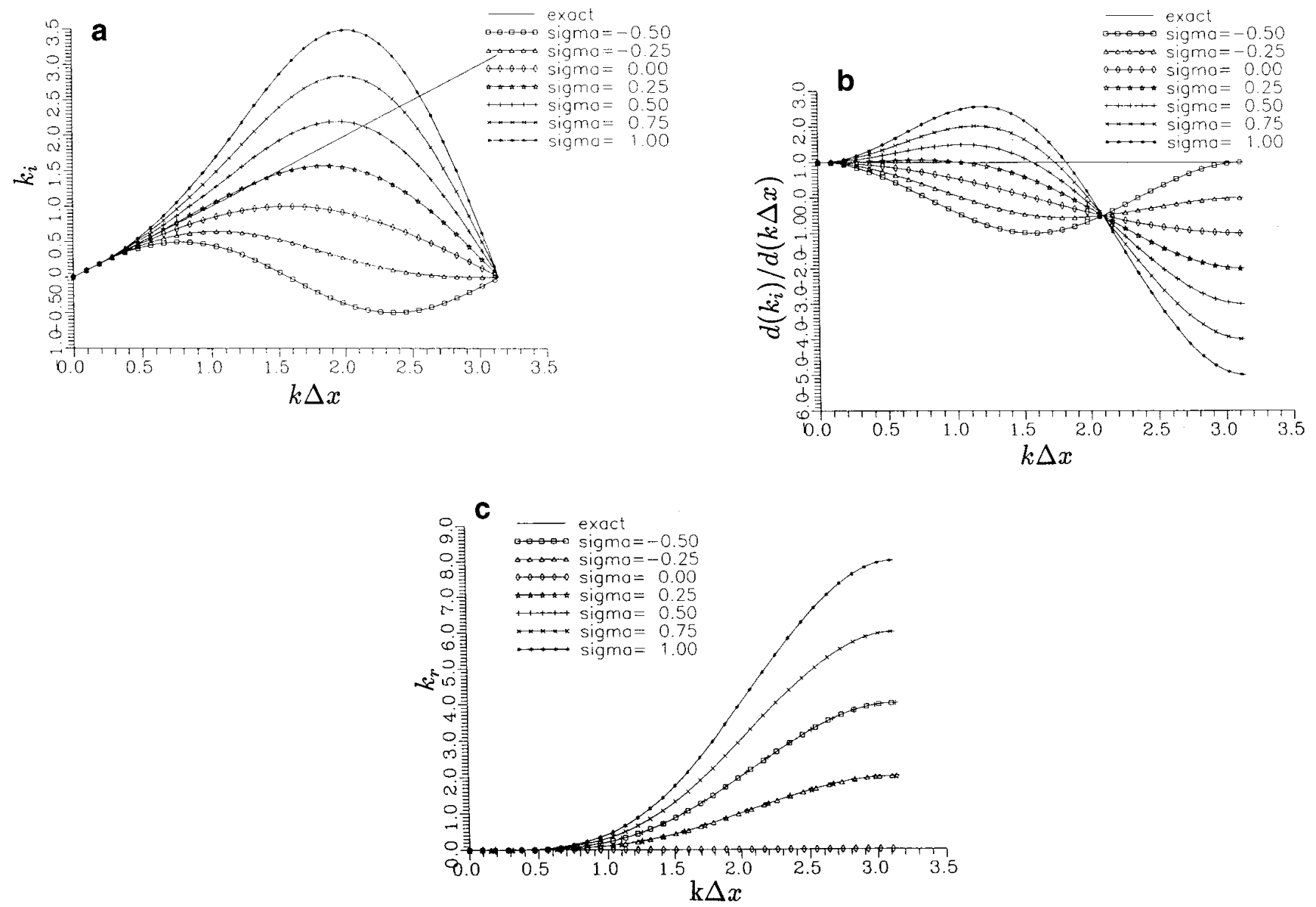

FIG. 3. a. Variation of $k_{i}$ with parameter $\sigma$ for $C N T$. b. Variation of $d\left(k_{i}\right) / d \alpha$ with parameter $\sigma$ for $C N T$. c. Variation of $k_{r}$ with parameter $\sigma$ for $C N T$.

where

$$
\tilde{\mu}=\left(\sum_{m} C_{m} \frac{\partial^{m-1} f}{\partial x^{m-1}} / \frac{\partial f}{\partial x}\right) c .
$$

Equation (2.19) is called the diffusion analogy equation and $\tilde{\mu}$ is called the diffusion analogy coefficient in [12]. $\tilde{\mu}$ is not the physical diffusion coefficient but it is useful to understand the properties of the numerical solutions. Defining,

$$
\begin{aligned}
\tilde{\mu}_{2} & =\left(\sum_{m} C_{2 m} \frac{\partial^{2 m-1} f}{\partial x^{2 m-1}} / \frac{\partial f}{\partial x}\right) \cdot c \\
\tilde{\mu}_{3} & =\left(\sum_{m} C_{2 m+1} \frac{\partial^{2 m} f}{\partial x^{2 m}} / \frac{\partial f}{\partial x}\right) \cdot c \\
a & =\left(\sum C_{2 m+1} \frac{\partial^{2 m} f}{\partial x^{2 m}}\right) / \frac{\partial^{2} u}{\partial x^{2}}
\end{aligned}
$$

we have

$$
\begin{gathered}
\tilde{\mu}=\tilde{\mu}_{2}+\tilde{\mu}_{3} \\
\tilde{\mu}_{3}=a \frac{\partial^{2} u}{\partial x^{2}} / \frac{\partial u}{\partial x} .
\end{gathered}
$$

The modified equation can also be written as

$$
\begin{aligned}
& \frac{\partial u}{\partial t}+\frac{\partial f}{\partial x}=\Delta x \frac{\partial}{\partial x}\left(\tilde{\mu}_{2} \frac{\partial u}{\partial x}\right)+\Delta x \frac{\partial}{\partial x}\left(\tilde{\mu}_{3} \frac{\partial u}{\partial x}\right) \\
& \frac{\partial u}{\partial t}+\frac{\partial f}{\partial x}=\Delta x \frac{\partial}{\partial x}\left(\tilde{\mu}_{2} \frac{\partial u}{\partial x}\right)+\Delta x \frac{\partial}{\partial x}\left(a \frac{\partial^{2} u}{\partial x^{2}}\right) .
\end{aligned}
$$

The coefficient $\tilde{\mu}_{2}$ is related with the dissipativity. The stability requires nonnegativity of the coefficient $\tilde{\mu}_{2}$. The coefficients $\tilde{\mu}_{3}$ and $a$ are related with modification of the phase speed and the group velocity of wavepackets. Equa- 

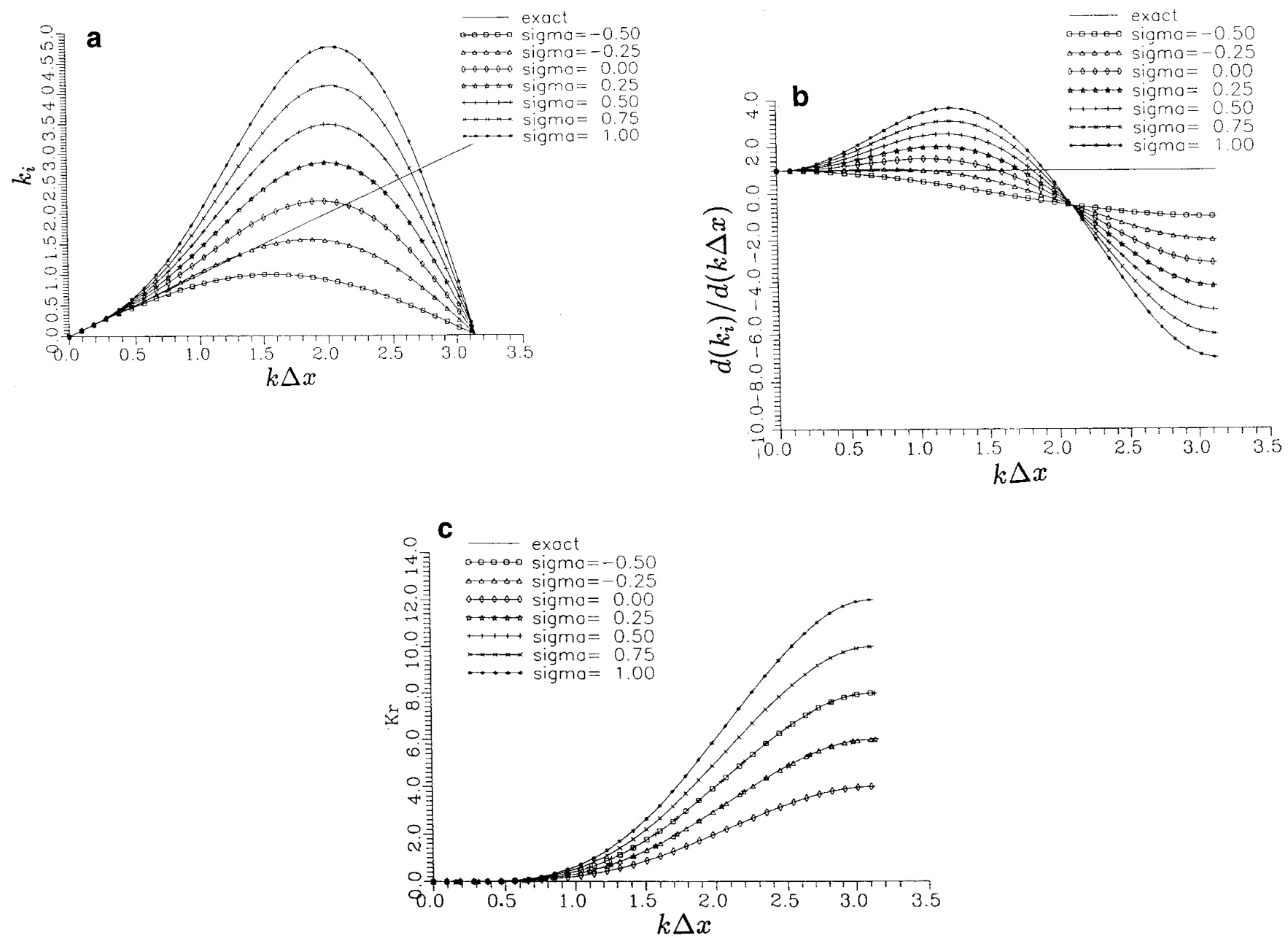

FIG. 4. a. Variation of $k_{i}$ with parameter $\sigma$ for $U P W$. b. Variation of $d\left(k_{i}\right) / d \alpha$ with parameter $\sigma$ for $U P W$. c. Variation of $k_{r}$ with parameter $\sigma$ for $U P W$.

tions (2.22) and (2.23) for the Fourier components $\exp (i k x)$ in the case $f_{u}=c$ can be expressed as

$$
\begin{aligned}
\tilde{\mu}_{2} & =\left(\sum_{m} C_{2 m}(-1)^{m-1} k^{2 m-2}\right) c \\
a & =\left(\sum_{m} C_{2 m+1}(-1)^{m-1} k^{2 m-2}\right) c .
\end{aligned}
$$

For improvement of the shock resolution the coefficient $\tilde{\mu}_{3}$ in (2.26) and $a$ in (2.27) must be controlled. As it was discussed before for improvement of the shock resolution the coefficient $a$ in (2.27) should be positive behind the shock and negative in front of the shock. Similar group velocity regulator defined in (2.17) can be used to control the coefficient $a$.

Now consider $\tilde{\mu}_{3}$ defined in (2.25). According to the sign of $\left(\partial^{2} u / \partial x^{2}\right) /(\partial u / \partial x)$ and requirement of $a$ for improvement of the shock resolution we have $\tilde{\mu}_{3}>0$ in both sides of the shock. $\tilde{\mu}_{3}>0$ also can be used to control the group velocity of the wavepackets in the numerical solutions.

In the present paper the group velocity of wavepackets for UCDA5 is controlled by the diffusion analogy coefficient as

$$
\frac{\partial u_{j}}{\partial t}+\frac{F_{j}}{\Delta x}=\frac{1}{\Delta x}\left(\bar{\mu}_{j+1 / 2} \delta_{x}^{+} u_{j}-\bar{\mu}_{j-1 / 2} \delta_{x}^{-} u_{j}\right)
$$

where

$$
\bar{\mu}=\bar{\mu}_{2}+\bar{\mu}_{3}
$$

and

$$
\begin{array}{ll}
\bar{\mu}_{2}=\sigma_{6} \Delta x^{6}\left|\partial^{5} f / \partial x^{5}\right||c| /(|\partial f / \partial x|+\varepsilon), & \sigma_{6}>0, \\
\bar{\mu}_{3}=\sigma_{7} \Delta x^{6}\left|\partial^{6} f / \partial x^{6}\right||c| /(|\partial f / \partial x|+\varepsilon), & \sigma_{7}>0 .
\end{array}
$$



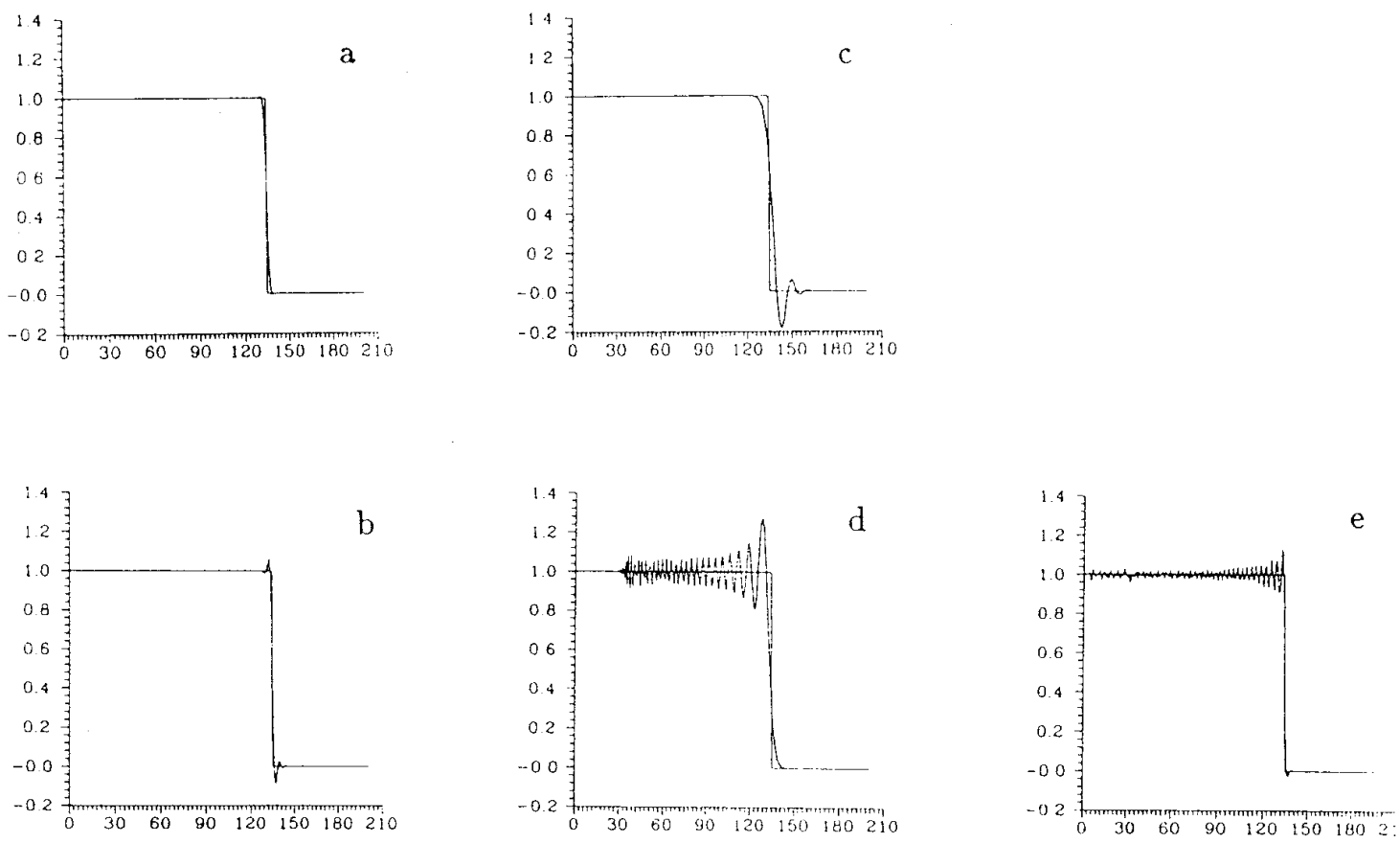

FIG. 5. Solution of the model equation at the time step $\mathrm{N}=500(c(\Delta t / \Delta x)=0.1, \Delta x=1 / 200)$ : a. 5th-order upwind compact scheme modified with the method of diffusion analogy; b. 5th-order upwind compact scheme; c. 2nd-order upwind scheme; d. 2nd-order centered scheme; e. 6thorder compact scheme.
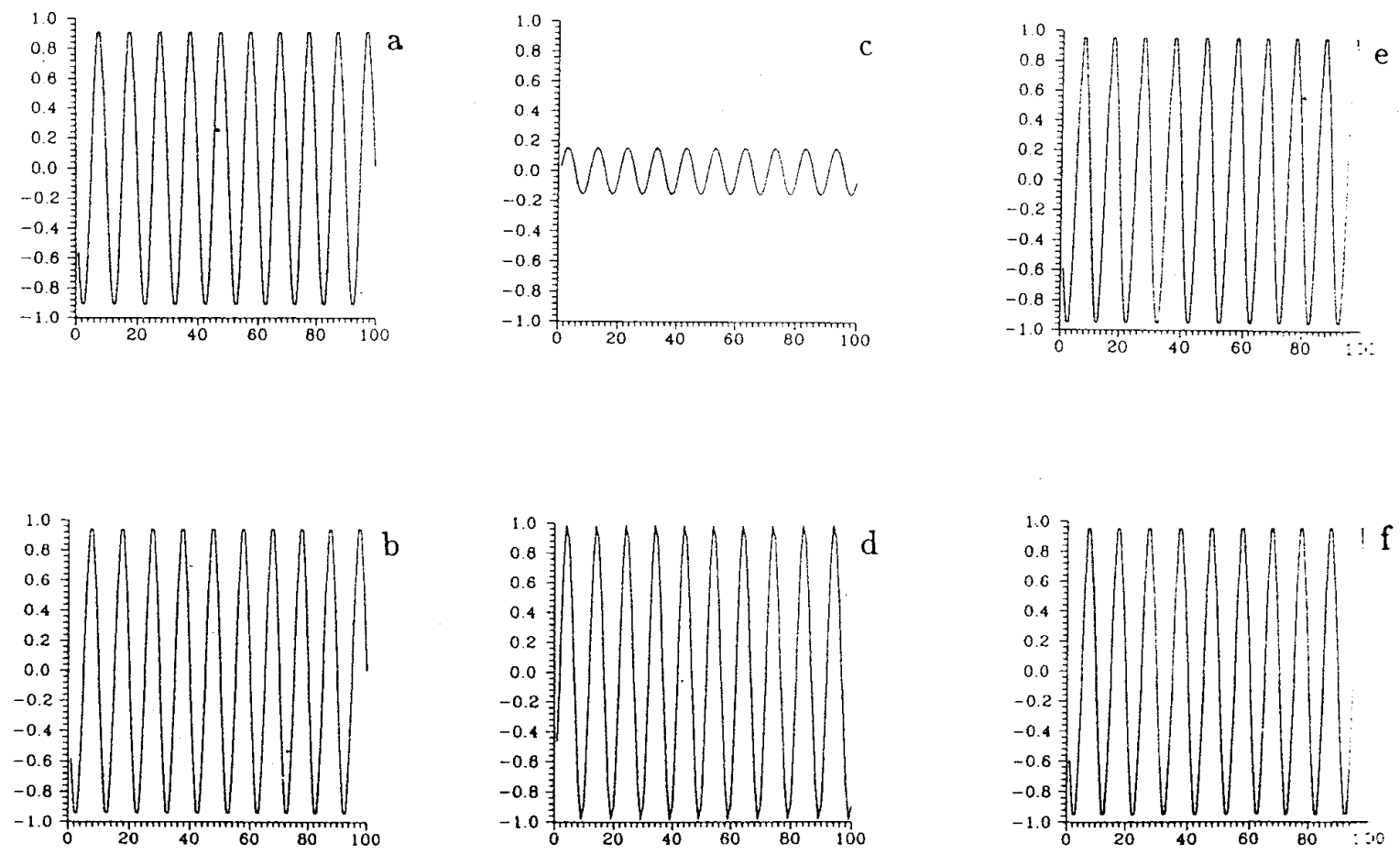

FIG. 6. Solution of the model equation at the time step $\mathrm{N}=500(c(\Delta t / \Delta x)=0.1, \Delta x=1 / 200)$ : a. 5 th-order upwind compact scheme modified with the method of diffusion analogy; b. 5th-order upwind compact scheme; c. 2nd-order upwind scheme; d. 2nd-order centered scheme; e. exact solution; f. 6th-order compact scheme. 
Introduction of $\varepsilon$ is to eliminate the singularity. In computation of the mixing layer $\sigma_{6}=\sigma_{7}=1 / 64$ is used. Introduction of $\bar{\mu}_{2}$ is to suppress the amplitudes of the oscillations with high wave numbers, and introduction of $\bar{\mu}_{3}$ is to control the group velocity so that the wavepackets move toward the moving shock. In computation the derivatives in (2.31) are approximated as

$\Delta x^{5}\left|\frac{\partial^{5} f^{ \pm}}{\partial x^{5}}\right|_{j+1 / 2}=\left|\delta_{x}^{4} F_{j+1}^{ \pm}+\delta_{x}^{4} F_{j}^{ \pm}\right| / 2$

$\Delta x^{6}\left|\frac{\partial^{6} f^{ \pm}}{\partial x^{6}}\right|_{j+1 / 2}=\left|\delta_{x}^{4} F_{j+1}^{ \pm}-\delta_{x}^{4} F_{j}^{ \pm}\right|$

$$
\delta_{x}^{4} F_{j}^{ \pm}=F_{j+2}^{+}-4 F_{j+1}^{+}+6 F_{j}^{ \pm}-4 F_{j-1}^{ \pm}+F_{j-2}^{ \pm}
$$

$$
\left|\frac{\partial f^{ \pm}}{\partial x}\right|_{j+1 / 2}=\left(\left|\frac{\partial f^{ \pm}}{\partial x}\right|_{j+1}+\left|\frac{\partial f^{ \pm}}{\partial x}\right|_{j}\right) / 2
$$$$
\Delta x\left|\frac{\partial f^{ \pm}}{\partial x}\right|_{j}=\frac{1}{16}\left(\left|F_{j+2}^{ \pm}\right|+4\left|F_{j+1}^{ \pm}\right|+6\left|F_{j}^{ \pm}\right|\right.
$$$$
\left.+4\left|F_{j-1}^{+}\right|+\left|F_{j-2}^{+}\right|\right)
$$

where $F_{j}^{ \pm}$is obtained from (2.3).

\section{DIFFERENCE APPROXIMATION OF THE N-S EQUATIONS}

\subsection{Governing Equations and \\ Coordinate Transformation}

The 2D dimensionless compressible $\mathrm{N}-\mathrm{S}$ equations for the perfect gas in the vector form can be written as

$$
\frac{\partial U}{\partial t}+\frac{\partial f_{1}}{\partial x}+\frac{\partial f_{2}}{\partial y}=\frac{\partial V_{1}}{\partial x}+\frac{\partial V_{2}}{\partial y}
$$

where the terms on the right-hand side are the viscous terms and $f_{1}$ and $f_{2}$ are the flux vectors corresponding to the $x$ and $y$ coordinate directions, respectively. For convenience rewrite (3.1) into a short form,

$$
\frac{\partial U}{\partial t}=L(U)
$$

In order to get fine solutions near the central line of the mixing layer the following coordinate transformation is used:

$$
\begin{aligned}
\tau & =t \\
\xi & =x \\
\eta & =\frac{1}{\tanh (b)} \tanh \left(\frac{b}{L_{y}} y\right) .
\end{aligned}
$$
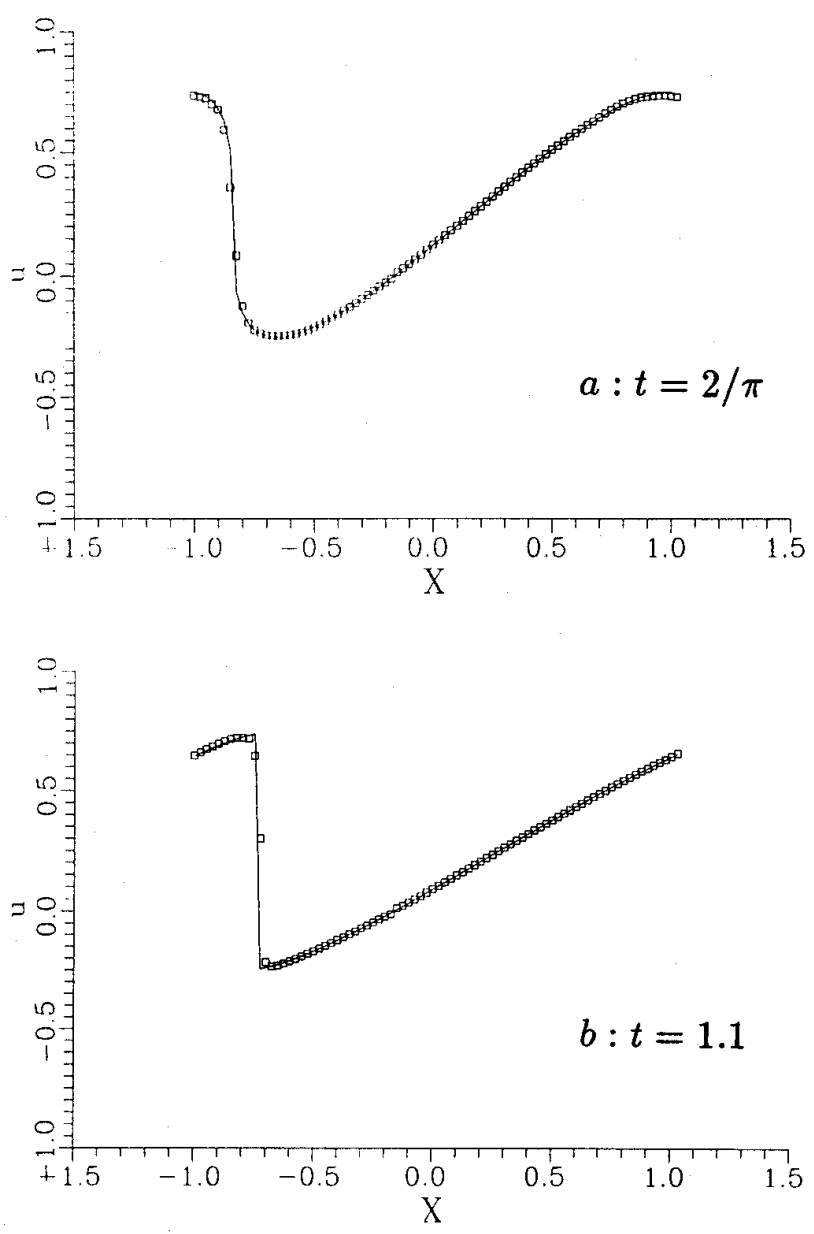

FIG. 7. Solution of the nonlinear Burgers equation with modified 2nd-order centered scheme: $a-t=2 / \pi ; b-t=1.1$.

Here $-L_{y}<y<L_{y}$ is the computational region in the normal direction, and $b$ is a stretch parameter.

\subsection{Difference Approximation}

As was mentioned the three-stage $\mathrm{R}-\mathrm{K}$ method is used in advance of the time. The second derivatives in the viscous terms are discretized by two times the application of sixth-order accurate compact difference approximation of the first derivative.

The flux vector splitting technique, $f_{1}=f_{1}^{+}+f_{1}^{-}, f_{2}=$ $f_{2}^{+}+f_{2}^{-}$, is used for the flux vectors [13]

$$
f_{\bar{k}}^{+}=A_{k}^{ \pm} U, \quad A_{k}^{ \pm}=S_{k}^{-1} \bigwedge_{\bar{k}}^{ \pm} S_{k}, \quad k=1,2,
$$

where $A_{k}$ is the Jacobian matrix corresponding to the flux vector $f_{k}, S_{k}$ is the matrix consisted of the left eigenvectors of the matrix $A_{k}, \wedge_{k}^{ \pm}$is the diagonal matrix with elements $\lambda_{k}^{ \pm}$with Buning-Steger regularization in computation of the mixing layer, and $\lambda_{k}$ are the eigenvalues of the matrix 

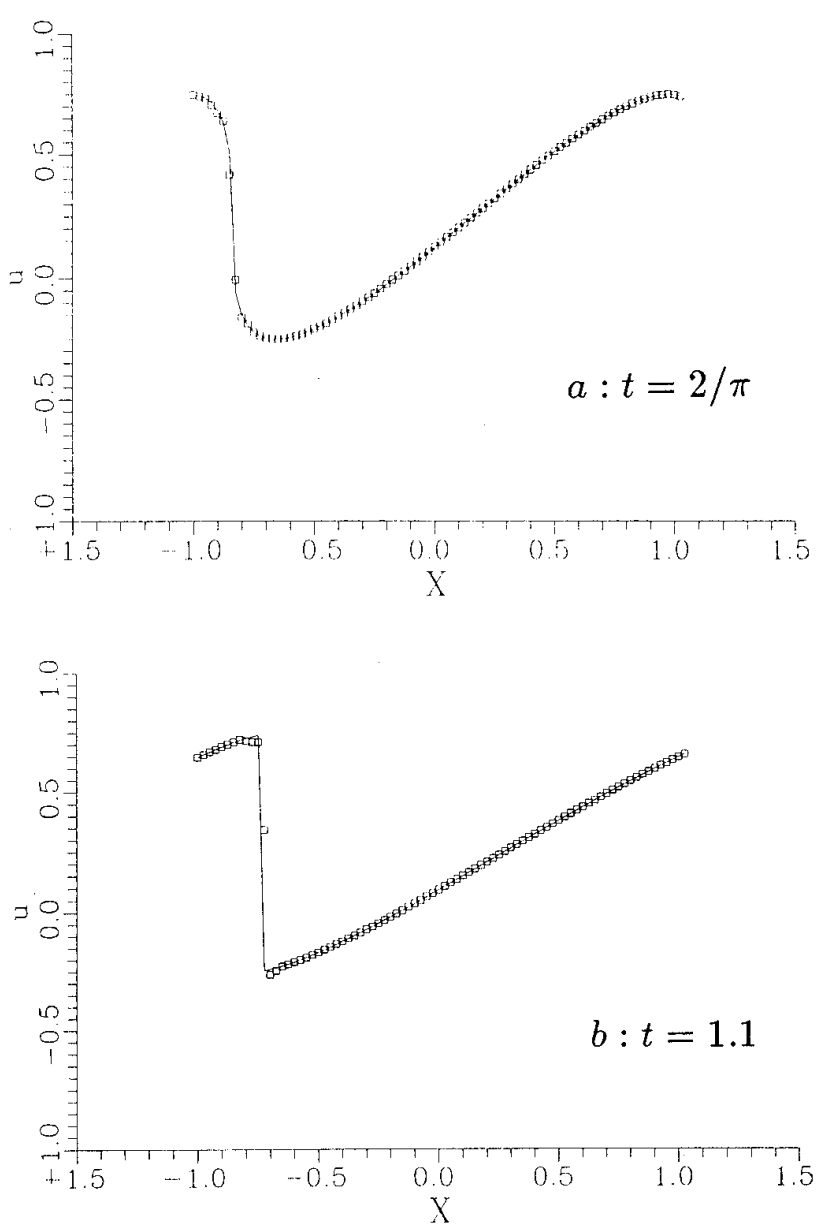

FIG. 8. Solution of the nonlinear Burgers equation with modified 4th-order centered scheme: $a-t=2 / \pi ; b-t=1.1$.

$A_{k}$. The split terms $(\partial / \partial x) f_{1}^{ \pm}$and $(\partial / \partial y) f_{2}^{ \pm}$are discretized with the fifth-order upwind compact approximation (2.3).

Periodic boundary conditions are used in the $x$ direction. Suppose the boundaries in the $y$ direction are located far away from the central line of the mixing layer. At these boundaries the flow is almost uniform and lower order accurate approximations are used.

At the points next to the boundaries in the $y$ direction the third-order upwind compact difference approximations for the convection terms are used [9],

$$
\frac{2}{3} F_{j}^{+}+\frac{1}{3} F_{j+1}^{+}=\frac{5}{6} \delta_{y}^{\mp} g_{j}+\frac{1}{6} \delta_{y}^{ \pm} g_{j},
$$

where $\delta_{y}^{-}$and $\delta_{y}^{+}$are 2-point backward and forward difference operators in the $y$ direction, respectively. In the computation of derivatives of the viscous terms at these points the following fourth-order compact difference approximation is used:

$$
\frac{1}{6} F_{j+1}+\frac{2}{3} F_{j}+\frac{1}{6} F_{j-1}=\delta_{y}^{0} g_{j}
$$

At boundary points in the $y$ direction second-order accurate difference approximations are used, for example, for the first component $f_{2,1}^{+}$of the split flux vector $f_{2}^{+}$at the lower boundary $\mathrm{j}=1$ we have

$$
\left.F_{j}\left(f_{2,1}^{+}\right)\right|_{j=1}=\left(\left.3 \delta_{y}^{+} f_{2,1}^{+}\right|_{j=1}-\left.\delta_{y}^{+} f_{2,1}^{+}\right|_{j=2}\right) / 2 .
$$

\subsection{Modification with the Method of Diffusion Analogy}

The group velocity of wavepackets is controlled with the method of diffusion analogy. According to the modified equations of the difference approximations of the $\mathrm{N}-\mathrm{S}$ equations we can get the following equations of diffusion analogy,

$$
\begin{gathered}
\frac{\partial U}{\partial t}=L(U)+D \\
D=D_{x}^{+}+D_{x}^{-}+D_{y}^{+}+D_{y}^{-},
\end{gathered}
$$

where $D_{x}^{+}, D_{x}^{-}, D_{y}^{+}$, and $D_{y}^{-}$have similar expressions and can be obtained easily, for example,
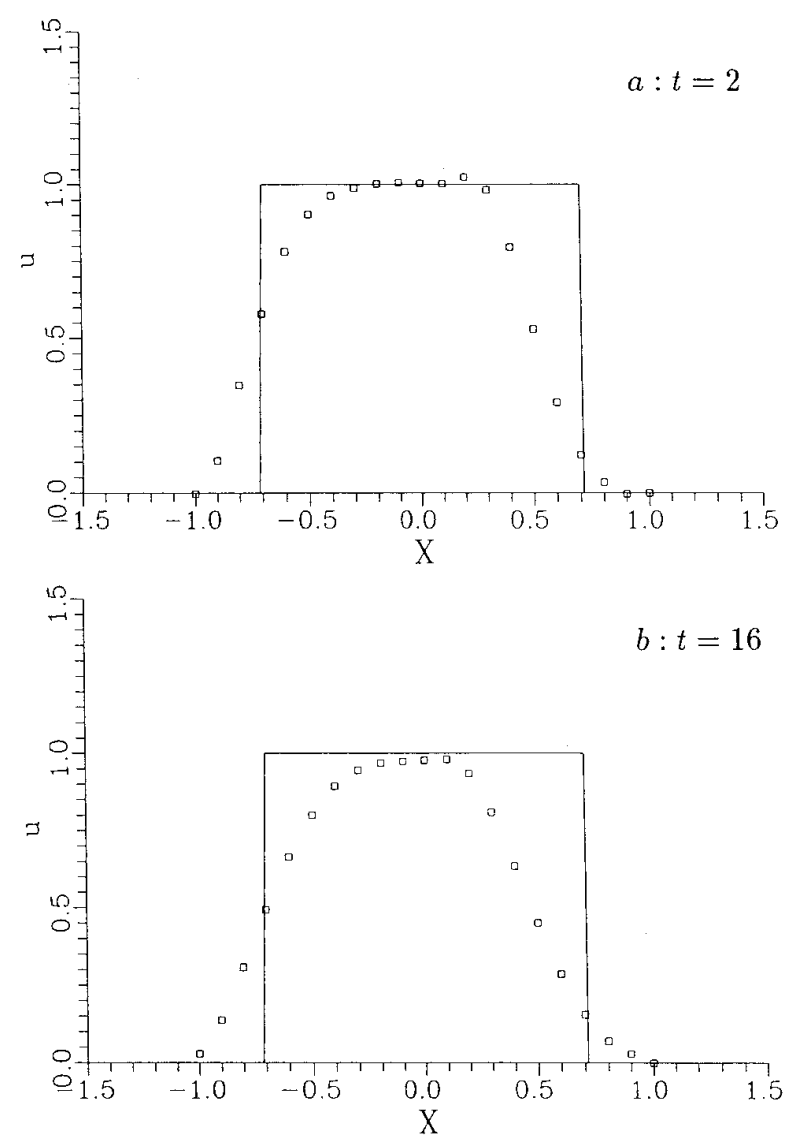

FIG. 9. Solution of $2 \mathrm{D}$ linear equation with modified 4 th-order centered scheme at $y=0: a-t=2 ; b-t=16$. 


$$
D_{x}^{+}=\Delta x \frac{\partial}{\partial x}\left[\left(M_{x, 2}^{+}+M_{x, 3}^{+}\right) A^{+} \frac{\partial U}{\partial x}\right]
$$

where $A^{+}$is the Jacobian matrix $\partial f_{1}^{+} / \partial U$ and $M_{x, 2}^{+}$and $M_{x, 3}^{+}$are diagonal matrices with elements

$$
\begin{aligned}
\left(M_{x, 2}^{+}\right)_{k} & =\sum_{m} C_{2 m} \frac{\partial^{2 m-1} f_{1, k}^{+}}{\partial x^{2 m-1}} / \frac{\partial f_{1, k}^{+}}{\partial x} \\
\left(M_{x, 3}^{+}\right)_{k} & =\sum_{m} C_{2 m+1} \frac{\partial^{2 m} f_{1, k}^{+}}{\partial x^{2 m}} / \frac{\partial f_{1, k}^{+}}{\partial x} .
\end{aligned}
$$

For the difference approximations of the $\mathrm{N}-\mathrm{S}$ equations with UCDA5 for the convection terms the equations

$$
\frac{\partial U}{\partial t}=L(U)+\bar{D}
$$

are used to reconstruct the difference approximations, where

$$
\begin{aligned}
\bar{D} & =\bar{D}_{x}^{+}+\bar{D}_{x}^{-}+\bar{D}_{y}^{+}+\bar{D}_{y}^{-}, \\
\bar{D}_{x}^{+} & =\Delta x \frac{\partial}{\partial x}\left[\left(\bar{M}_{x, 2}^{+}+\bar{M}_{x, 3}^{+}\right)\left|A_{1}^{+}\right| \frac{\partial U}{\partial x}\right] .
\end{aligned}
$$

$\left(\bar{M}_{x, 2}^{+}+\bar{M}_{x, 3}^{+}\right)$are diagonal matrices with the elements

$$
\left(\bar{M}_{x, 2}^{+}+\bar{M}_{x, 3}^{+}\right)_{k}=\Delta x^{6}\left\{\frac{\sigma_{6}\left|\partial^{5} f_{1, k}^{+} / \partial x^{5}\right|+\sigma_{7}\left|\partial^{6} f_{1, k}^{+} / \partial x^{6}\right|}{\left|\partial f_{1, k}^{+} / \partial x\right|+\varepsilon}\right\}
$$

where $\sigma_{6}$ and $\sigma_{7}$ are positive constant parameters as in (2.31). The derivatives for each component $f_{1, k}^{+}$of the split flux vector $f_{1}^{+}$are approximated as for the scalar case analyzed in Subsection 2.3. For obtaining (3.11) only the leading terms in (3.9) are used. For simplicity of computation, the matrix $\left|A^{+}\right|$is replaced by a diagonal matrix with elements $|u|+c$. Similar expressions for $\bar{D}_{x}^{-}, \bar{D}_{y}^{+}$, and $\bar{D}_{y}^{-}$can be obtained easily.

\section{NUMERICAL EXAMPLES FOR MODEL EQUATIONS}

EXAMPLE 1. The above-presented method with control of group velocity, combined with the three stage RungeKutta type TVD time discretization [11], is used to solve the linear simple wave equation

$$
\frac{\partial u}{\partial t}+\frac{\partial u}{\partial x}=0
$$

Results with the initial conditions

$$
u(x, 0)=\left\{\begin{array}{l}
1, \quad 0 \leq x<\frac{5}{12} \\
0, \quad \frac{5}{12} \leq x \leq 1
\end{array}\right.
$$

at time step $N=500(\Delta t / \Delta x=0.1, \Delta x=1 / 200)$ are given in Fig. 5. It can be seen that the oscillations are behind the shock for the symmetrical difference approximations because of the slower group velocity of wavepackets for all wave numbers (Figs. 5d, e). For the second-order upwind scheme (Fig. 5c) the oscillations are produced in front of the shock because of the higher group velocity for the wavepackets with moderate wave numbers (see Fig. $4 \mathrm{~b}$ for $\sigma=0)$. The oscillations are not noted behind the shock because of dissipation. For the UCDA5 the oscillations with small amplitudes are on both sides of the shock. The small amplitude is because of dissipation of the scheme for the moderate and high wave numbers. The oscillations on both sides of the shock are caused by higher group velocity of the wavepackets with moderate wave numbers and slower group velocity of the wavepackets with high wave numbers. Figure 5a shows the results obtained with modified UCDA5 expressed in (2.30) and (2.31). It can be seen that the shock resolution is much improved. In Fig. 6 results with the same schemes are shown at step $N=$ 500 with the periodic initial conditions

$$
\sin (x, 0)=\sin (2 \pi k x), \quad k=10,0 \leq x \leq 1 .
$$

From Fig. 6 it can be seen that the dissipation of the threepoint second-order upwind scheme is too large (see Fig. $6 c$ ). From Figs. 6c and d compared with the exact solution in Fig. 6e near the point $x=0$ it can be seen that the phase errors are very large for the low accurate schemes. With modification of the scheme the shock resolution is improved (Fig. 5a) but new truncation error is introduced. From Fig. 6a, compared with the exact solution Fig. 6e near $x=0$, it can be seen that the influence of the newly introduced error on the phase speed is negligible.

EXAMPLE 2. The second- and fourth-order centered difference approximations modified with the method of diffusion analogy, combined with three-stage $\mathrm{R}-\mathrm{K}$ time discretizations, are used to solve the nonlinear Burgers equation with periodic initial conditions [11],

$$
\begin{aligned}
\frac{\partial u}{\partial t}+\frac{\partial}{\partial x}\left(\frac{u^{2}}{2}\right) & =0, & & -1 \leq x \leq 1, \\
u(x, 0) & =\frac{1}{4}+\frac{1}{2} \sin (\pi x), & & -1 \leq x \leq 1 .
\end{aligned}
$$



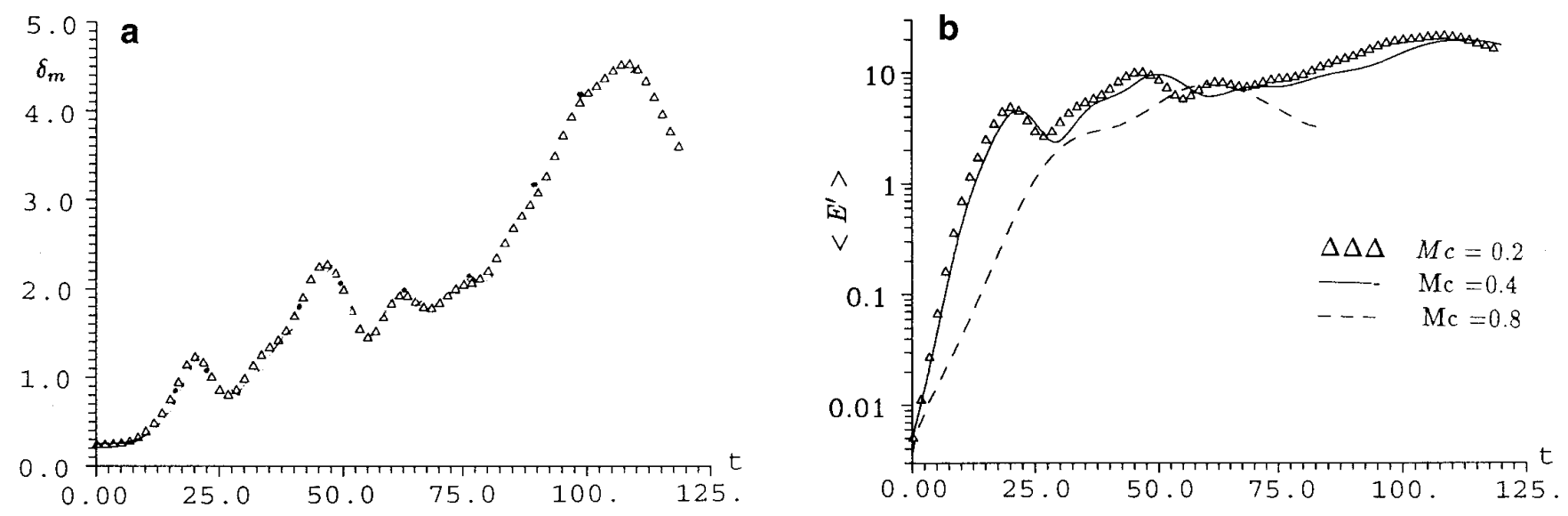

FIG. 10. a. Variation of $\delta_{m}$ vs time $t$ for $\mathrm{Mc}=0.2, \ldots$, is obtained from [16]. b. Variation of $\left\langle E^{\prime}\right\rangle$ vs time $t$ for different Mc.

The exact solution is smooth up to $t=2 / \pi$; then it develops a moving shock which interacts with the rarefaction waves. Figure 7 shows results with the modified second-order accurate centered scheme at $t=2 / \pi$ and $t=1.1$. In computations the parameter $\sigma_{0}=0.5$ in (2.17) is used. Figure 8 shows the results with modified fourth-order centered scheme with $\sigma_{0}=0.1$ at $t=2 / \pi$ and $t=1$.1. From the figures it can be seen that the shock solutions are improved with the newly presented method which is much simpler than the method in [11].

ExAmple 3. We use the same schemes as in Example 2 to solve a linear problem

$$
\begin{aligned}
& \frac{\partial u}{\partial t}+\frac{\partial u}{\partial x}+\frac{\partial u}{\partial y}=0, \quad-1 \leq x, y \leq 1, \\
& u(x, y, 0)= \begin{cases}1, & \text { if }(x, y) \in S, \\
0, & \text { if }(x, y) \in S,\end{cases}
\end{aligned}
$$

where $S=\{(x, y):|x-y|<1 / \sqrt{2},|x+y|<1 / \sqrt{2}\}$ is a unit square centered at the origin and rotated by an angle of $\pi / 4$ (seen $[11,18]$ ). This example is used to study the amount of smearing of contact discontinuities of the method. Periodic conditions are used at the boundaries of the computational region. The computed results with the modified fourth-order centered scheme are given in Fig. 9 for $y=0$ at $t=2$ (after one period in time) and $t=16$ (after eight periods in time). In computations $\sigma_{0}=0.05$ and $\Delta x=0.1$ are used. From the figures we see asymmetry of the solution as in [11]. The smearing of the contact discontinuities is a little larger than 4-4-LF-ENO in [11]. This is because of the reduction of accuracy of the method near the extremal points. It can be improved with large $\varepsilon$ in $(2.31)$.

\section{NUMERICAL SIMULATION OF COMPRESSIBLE MIXING LAYERS}

\subsection{Initial and Boundary Conditions}

The temporally growing compressible mixing layers are simulated numerically. The initial conditions consist

TABLE 1

\begin{tabular}{|c|c|c|c|c|}
\hline \multirow[b]{2}{*}{$\begin{array}{l}\text { Characteristics } \\
\text { time }\end{array}$} & \multicolumn{2}{|c|}{$2 \mathrm{D}$} & \multicolumn{2}{|c|}{$3 \mathrm{D}$} \\
\hline & Incompressible & $\begin{array}{c}\mathrm{M}_{\infty}=0.2, \mathrm{Re}=400, \\
\text { three pairing }\end{array}$ & Incompressible & $\begin{array}{c}\mathrm{M}_{\infty}=0.2, \mathrm{Re}=400, \\
\text { one pairing }\end{array}$ \\
\hline$T_{p 1}$ & 21.5 & 20.17 & 21.5 & 21.6 \\
\hline$T_{p 2}$ & 47.0 & 46.17 & & \\
\hline$T_{p 3}$ & 103.7 & 107.81 & & \\
\hline$T_{s 1}$ & 32.0 & 32.66 & & \\
\hline$T_{0}$ & 66.7 & 66.33 & & \\
\hline
\end{tabular}

Characteristic Time $T_{\mathrm{np}}$ and $T_{\mathrm{ns}}$

Note. $T_{\mathrm{np}}$, the nth pairing time of the rollers; $T_{\mathrm{ns}}$, the time at which the vorticity from the spiral arms comes back into the braid region. 

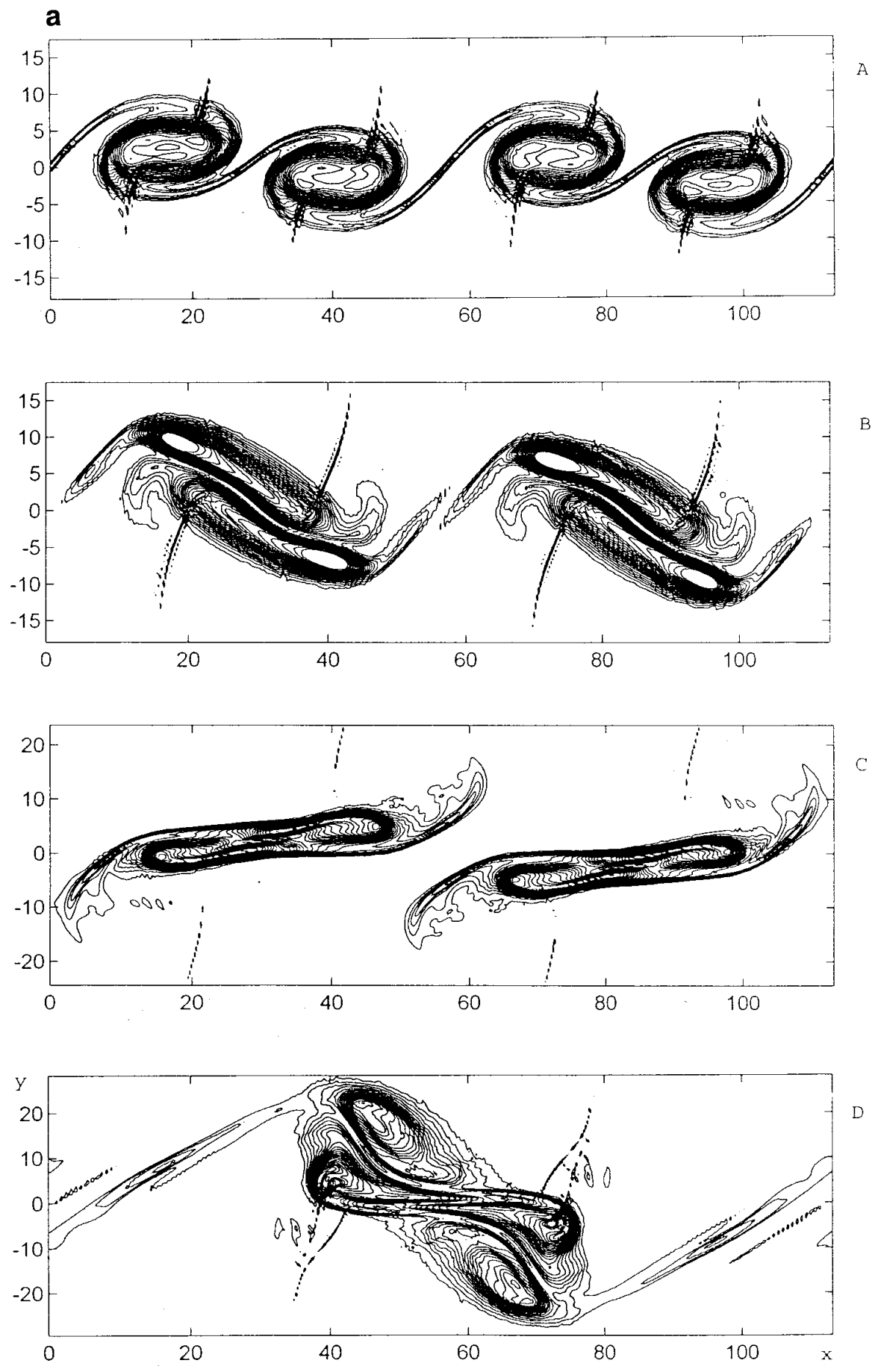

FIG. 11. a. Vorticity contours for $\mathrm{Mc}=0.8$ at different time: $\mathrm{A}-\mathrm{T}=32.00, \omega_{\min }=-0.50, \omega_{\max }=-0.026, \Delta \omega=0.020 ; \mathrm{B}-48.00, \omega_{\min }=-0.50$, $\omega_{\max }=-0.026, \Delta \omega=0.020 ; \mathrm{C}-72.00, \omega_{\min }=-0.60, \omega_{\max }=-0.026, \Delta \omega=0.024 ; \mathrm{D}-111.94, \omega_{\min }=-0.40, \omega_{\max }=-0.026, \Delta \omega=0.016 . \mathrm{b} . \mathrm{Density}$ contours for $\mathrm{Mc}=0.8$ at different time: $\mathrm{A}-\mathrm{T}=32.00, \mathrm{~B}-\mathrm{T}=48.00, \mathrm{C}-\mathrm{T}=72.00, \mathrm{D}-\mathrm{T}=111.94$.

of a parallel mean flow with a hyperbolic tangent profile plus perturbation of the velocity components. The mean profile of the velocity is specified by the relations

$$
\begin{aligned}
& \bar{u}=A[B+\tanh (\beta y)], \quad \beta>0, \\
& \bar{v}=0 \\
& A=\frac{u_{1}-u_{2}}{2}, \quad B=\frac{u_{1}+u_{2}}{2},
\end{aligned}
$$



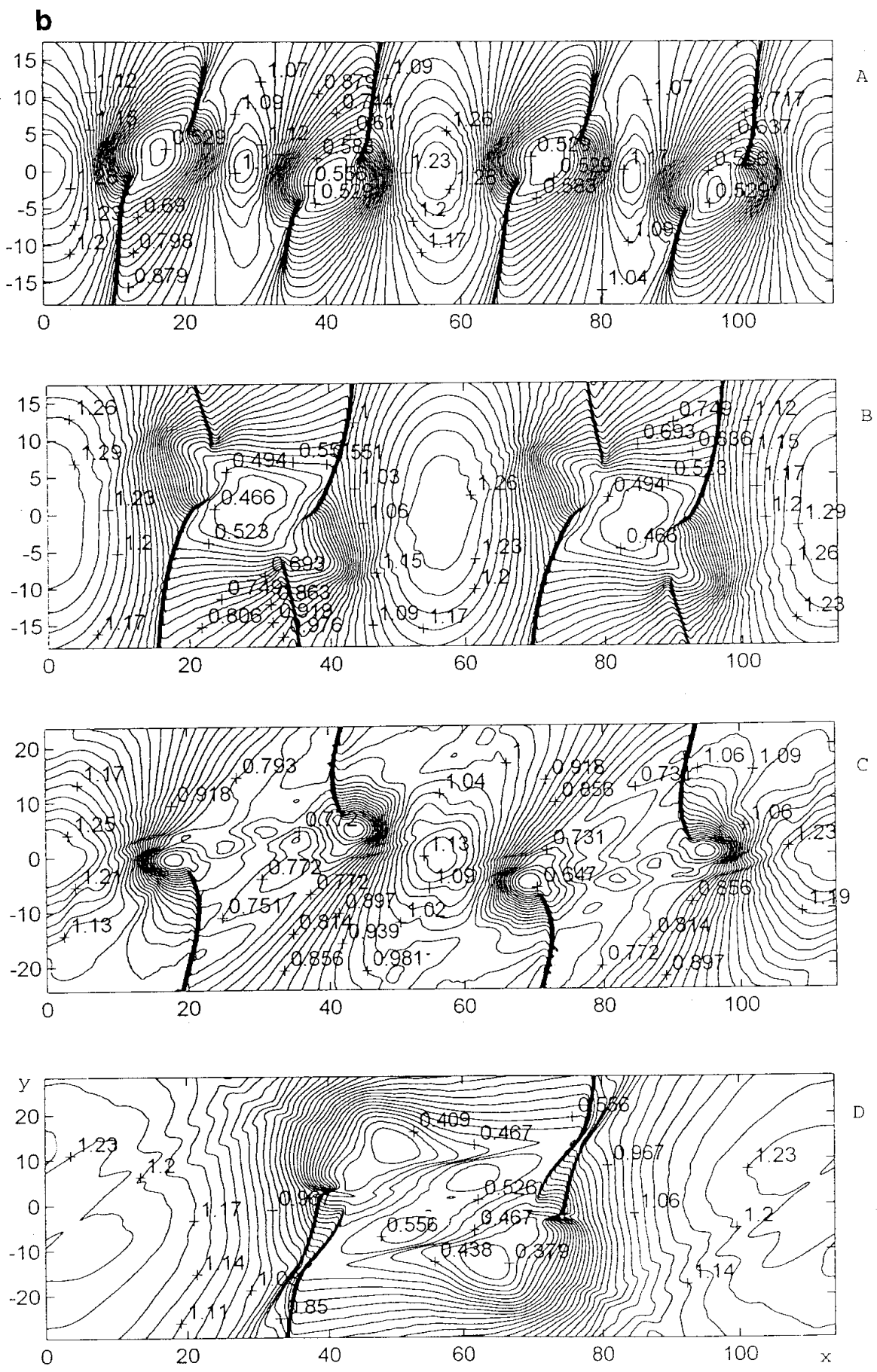

FIG. 11-Continued

where $u_{1}$ and $u_{2}$ are the upper and lower uniform streamwise velocity components respectively. In the present computation $u_{1}=-u_{2}=1$ and $\beta=1$ are used. With the Prandtl number of unity the mean temperature profile is specified as

$$
\bar{T}=1+M_{1}^{2} \frac{\gamma-1}{2}\left(1-\bar{u}^{2}\right)
$$

where $M_{1}$ is the Mach number corresponding to the upper far flow field. $\bar{T}=1$ is also used for the case with small 
convective Mach number Mc which is defined by the relation [14]

$$
\mathrm{Mc}=\left(u_{1}-u_{2}\right) /\left(c_{1}+c_{2}\right)
$$

where $c_{1}$ and $c_{2}$ are the sound speed in the upper and lower far flow field, respectively. In the present computation $c_{1}=c_{2}$ and $M_{1}=M_{2}$. Pressure is assumed uniform initially so the mean density profile can be obtained directly from the temperature profile. Sutherland's law is used to compute the viscosity coefficient. The Reynolds number is based on half the initial vorticity thickness of the mixing layer, half the velocity difference across the layer, and the free-stream viscosity.

The measure of the width of the mixing layer is the vorticity thickness which is computed based on the massweighted velocity profile

$$
\delta_{\omega}(t)=\frac{u_{1}-u_{2}}{|(d / d y)(\overline{\rho u} / \bar{\rho})|_{\max }}
$$

where $\bar{\rho}$ and $\overline{\rho u}$ are computed using integral spatial average in the $x$ direction defined by

$$
\bar{\varphi}(y)=\frac{1}{L_{x}} \int_{0}^{L x} \varphi(x, y) d x, \quad \varphi=\rho, \rho u
$$

$0 \leq x \leq L_{x}$ is the computational region in the $x$ direction.

The initial perturbation of the velocity components are described by

$$
\begin{aligned}
& u^{\prime}=\sum_{j} \varepsilon_{j}\left\{\phi_{\alpha_{j}, r}^{\prime}(y) \cos \left(\alpha_{j} x\right)-\phi_{\alpha_{j}, i}^{\prime}(y) \sin \left(\alpha_{j} x\right)\right\} \\
& v^{\prime}=\sum_{j} \varepsilon_{j}\left\{\phi_{\alpha_{j}, r}(y) \sin \left(\alpha_{j} x\right)-\phi_{\alpha_{j}, i}(y) \cos \left(\alpha_{j} x\right)\right\} \alpha_{j},
\end{aligned}
$$

where the wave number $\alpha_{1}=0.4446$ corresponds to the most unstable mode obtained from the linear inviscid flow analysis given by Michalke [15]. $\phi_{\alpha_{1}, r}$ and $\phi_{\alpha_{1}, i}$ are the real and imaginary parts of the corresponding eigenfunction for the stream function. $\phi_{\alpha_{1}, r}^{\prime}$ and $\phi_{\alpha_{1}, i}^{\prime}$ are derivatives of $\phi_{\alpha_{1}, r}$ and $\phi_{\alpha_{1}, i}$ with respect to $y . \phi_{\alpha 2, r}$ and $\phi_{\alpha 2, i}$ are the eigenfunctions corresponding to the wave number $\alpha_{2}$, and $\phi_{\alpha 2, r}^{\prime}, \phi_{\alpha 2, i}^{\prime}$ are their first derivatives. $\phi_{\alpha_{i}, r}$ and $\phi_{\alpha_{i}, i}$ are normalized. The coefficients $\varepsilon_{1}=\varepsilon_{2}=\varepsilon_{3}=\varepsilon_{4}=0.05$ are used for the case of three-pairing. For formation of three-pairing the following wave numbers are chosen

$$
\alpha_{1}=0.4446, \quad \alpha_{2}=\frac{1}{2} \alpha_{1}, \quad \alpha_{3}=\frac{1}{4} \alpha_{1}, \quad \alpha_{4}=\frac{1}{8} \alpha_{1} .
$$

The domain of computation is $0 \leq x \leq 16 \pi / \alpha_{1},-12 \pi / \alpha_{1}$ $\leq y \leq 12 \pi / \alpha_{1}$. The purpose of the paper is to consider the applicability of the newly developed method for capturing the shock and the small structures in the flow fields. The disturbances are not added to the static pressure and temperature. The flow is unstable to small disturbances.

The fluctuation of a function $\varphi$ is defined as

$$
\varphi^{\prime}(x, y)=\varphi(x, y)-\bar{\varphi}(y),
$$

where $\bar{\varphi}$ is obtained from (5.5). The fluctuating kinetic energy is defined as

$$
E_{c}^{\prime}(x, y)=\frac{1}{2}\left[\left(\frac{\overline{\rho u^{\prime}}}{\bar{\rho}}\right)^{2}+\left(\frac{\overline{\rho v^{\prime}}}{\bar{\rho}}\right)^{2}\right]
$$

and its global amplitude is defined as

$$
\left\langle E_{c}^{\prime}\right\rangle=\frac{1}{L_{x} L_{y}} \int_{L_{x} L_{y}} E_{c}^{\prime}(x, y) d x d y .
$$

The mesh grid system is $I N \times J N=200 \times 200$ for $\mathrm{Mc}=$ 0.2 , and $I N \times J N=502 \times 156$ for the case $\mathrm{Mc}=0.8$. The UCDA5 with modification mentioned in Subsections 3.2 and 3.3 is used to approximate the convective terms in the $\mathrm{N}-\mathrm{S}$ equations, the sixth-order compact difference approximation is used for the viscous terms, and the three-stage $\mathrm{R}-\mathrm{K}$ time discretization is used to advance in time.

\subsection{Computation for Convective Mach Number $M c=0.2$}

For validation of the method the case $\mathrm{Mc}=0.2$ with three-pairing is computed in order to compare with the results of incompressible mixing layers. With these initial conditions the flow loses its stability and eight orderly located rollers are soon formed. After a while the thickness of the viscous layers enlarges and the growth of the perturbation of the first subharmonic becomes obvious. The effect of nonlinearity leads to vortex pairing. The variations of the moment thickness $\delta_{m}$ for Mc $=0.2$, and the global amplitude of the fluctuation kinetic energy $\left\langle E_{c}^{\prime}\right\rangle$ versus time $t$ are given in Fig. 10 from which it can be seen that the growth rate decreases with increasing the convection Mach number. From Fig. 10a we also can see that the variation of the moment thickness for $\mathrm{Mc}=0.2$ agrees well with the results in [16]. For comparison, in Table 1 the characteristic time $T_{\mathrm{np}}$ and $T_{\mathrm{ns}}$, obtained in the present computation, are given ( $T_{\mathrm{np}}$ is the $n$th pairing time of the rollers and $T_{\mathrm{ns}}$ is the time at which the vorticity from the spiral arms reenter the surviving braid region after the nth pairing [16]). In the table are also given the corresponding results for the incompressible mixing layers in [16]. We see that they agree well with each other. From the above-presented results it can be seen that the flow characteristics for the compressible mixing layers with small Mach number, $\mathrm{Mc}=0.2$, are basically similar to those for the incompressible mixing layers. 


\subsection{Computation for Convective Mach Number} $M c=0.8$

Experiments show that the growth rate of the mixing layer decreases with increasing the convective Mach number. The same trend is obtained from the present numerical simulation (see Fig. 10b). The vorticity and density contours for $\mathrm{Mc}=0.8$ at different characteristic times are given in Fig. 11. From these figures we see that the flow field structures for $\mathrm{Mc}=0.8$ are different from those for the smaller Mc due to compressibility. In the process of pairing the shocks change their intensity and shape. As the number of rollers the number of shocks is halved at each pairing (see Fig. 11b). In Fig. 12 the streamwise variations of the pressure and the local Mach number at the time of third pairing along $y=11.2$ are given (near the upper-left vortex center shown in D of Fig. 11a). From the figure we see decreasing the pressure in the expansion region, the pressure plateau near the upper-left vortex center, and suddenly increasing the pressure across the shock. The local Mach number before the shock is about 1.4. The strength (change in pressure divided by the initial pressure) at the time of third pairing $t=112$ is equal to 0.97. From the complex flow field with shock structures it can be seen that high resolution of unstationary shocks can be obtained using the newly developed high order accurate method.

\section{CONCLUSION}

A fifth-order accurate upwind compact scheme with diffusion analogy modification is developed. The reason for oscillation production in the numerical solutions is analysed heuristically. It is because of a nonuniform group velocity of wavepackets. The oscillations in the numerical solutions are produced behind the shock if the group velocity of wavepackets with moderate and high wave numbers in the numerical solutions is slower than the group velocity of the exact solution. The oscillations are produced in front of the shock if the group velocity of the wave packets with moderate and high wave numbers is faster than the group velocity of the exact solution. The method of diffusion analogy can be used to control the group velocity of wavepackets so that the Fourier components in the numerical solutions have a faster group velocity of wavepackets behind the shock and a slower group velocity in front of the shock. The fifth-order accurate upwind compact scheme reconstructed with the method of diffusion analogy is used

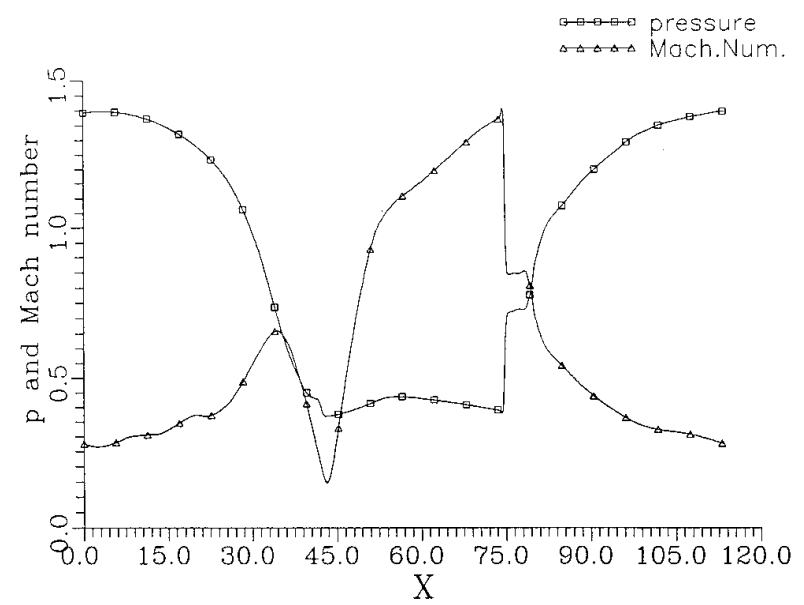

FIG. 12. Streamwise variations of the pressure and Mach number at $y=11.2$ at the time of third pairing.

to simulate the compressible mixing layers. The new method is useful for both capturing the shocks and the flow fields with small scale structures.

\section{ACKNOWLEDGMENTS}

This work is supported by the National Natural Science Foundation of China and the National Basic Research Project.

\section{REFERENCES}

1. S. K. Lele, AIAA Paper 89-0374, 1989.

2. S. Lee, S. K. Lele, and P. Moin, Phys. Fluids A3(4) (1991).

3. N. D. Sandham and W. C. Reynolds, J. Fluid Mech. 224, 133 (1991).

4. D. Papamoschou and A. Roshko, J. Fluid Mech. 197, 453 (1988).

5. D. W. Bogdanoff, AIAA J. 21(6) 926 (1983).

6. S. A. Ragab and J. L. Wu, Phys. Fluids A1(6), 957 (1989).

7. N. D. Sandham and W. C. Reynolds, AIAA J. 28(4), 618 (1990).

8. C. K. W. Tam and F. Q. Hu, J. Fluid Mech. 203, 51 (1986).

9. D. Fu, Y. Ma, and H. Liu, in Proceedings, 5th International Symposium on CFD, Sendai, 1993, Vol. 1, p. 184.

10. S. K. Lele, J. Comput. Phys. 103, 16 (1992).

11. Shu Chi-Wang and Osher, S., J. Comput. Phys. 77, 439 (1988).

12. Y. Ma and D. Fu, Sci. China Ser. A 35(9), 1090 (1992).

13. J. L. Steger and R. F. Warming, NASA TM D-78605.

14. D. Papamoschou and A. Roshko, AIAA Paper 86-0162, 1986.

15. A. Michalke, J. Fluid Mech. 19(4) 543 (1964).

16. R. D. Moser and M. M. Rogers, J. Fluid Mech., 247, 265 (1993).

17. L. Trefethen, SIAM Rev. 24(2), 136.

18. A. Harten, in Proceedings, International Conference on Hyperbolic Problems, Saint-Etieme, 1986. 